\title{
Acute and Long-term Outcomes of Cryoballoon Ablation in Patients with Atrial Fibrillation. Primary Results of the Spanish Cryoballoon Ablation Registry (RECABA)
}

Ángel Ferrero Loma-Osorio ( $\square$ angelferrero@hotmail.com )

Hospital Clínico Universitario. INCLIVA Foundation

Rocío Cózar

Hospital Universitario Virgen Macarena

Arcadio García-Alberola

Hospital Universitario Virgen de la Arrixaca - IMIB. Murcia

Ermengol Valles

Hospital del Mar. Barcelona

Alberto Barrera

Hospital Virgen de la Victoria Málaga

Jorge Toquero

Hospital Universitario Puerta de Hierro

Jose Miguel Ormaetxe

Hospital de Basurto

Juan Martínez Sánchez

Hospital Universitario Virgen de la Arrixaca - IMIB. Murcia

Ricardo Ruiz-Granell

Hospital Clínico Universitario. INCLIVA Foundation

Pablo Bastos Amador

Hospital Universitario Virgen Macarena

Jose Manuel Rubio

Hospital Universitario Fundación Jiménez Díaz

Julio Martí-Amor

Hospital del Mar. Barcelona

Patricia Pascual

Medtronic Iberia, S.A

Irene Molina

Medtronic Iberia, S.A

Jesús Daniel Martínez-Alday

Hospital de Basurto 


\section{Research Article}

Keywords: Spanish Registry, Cryoballoon, Atrial Fibrillation, Ablation.

Posted Date: April 22nd, 2021

DOI: https://doi.org/10.21203/rs.3.rs-440392/v1

License: (c) (1) This work is licensed under a Creative Commons Attribution 4.0 International License. Read Full License 


\section{Abstract \\ Background}

Cryoablation is safe and effective for the treatment of atrial fibrillation (AF) in controlled clinical trials, but contemporary real-world usage and outcomes are limited.

\section{Objectives}

The Report of the Spanish Cryoballoon Ablation Registry (RECABA) was designed to evaluate acute and 12-month outcomes of cryoballoon ablation for the treatment of AF in Spain.

\section{Methods}

Patients from 27 Spanish centers were prospectively enrolled. Patients were treated with cryoballoon ablation and managed according to standard of care protocols at each center. Theprimary endpoint was $\geq 30 \mathrm{sec}$ freedom from AF at 12-mo after a 3-mo blanking period. Secondary endpoints included a description of patient characteristics, cryoablation procedural strategy and safety, and predictors of efficacy.

\section{Results}

In total, 1742 patients $(71.4 \%$ PAF, $68.8 \%$ male, mean age $58.02 \pm 10.40$ years, $76.1 \%$ overweight or obese, $\mathrm{CHA}_{2} \mathrm{DS}_{2}$-VASc index $1.40 \pm 1.28$ ) were enrolled. Patients received $7.2 \pm 2.67$ cryoapplications. PV potentials could be detected in $61 \%$ of the PVs during ablation, with a mean time to block of $52.9 \pm 37.02$ seconds. Acute PVI was observed in $97 \%$ of PVs with $75.8 \%$ isolated with the first cryoapplication. Mean procedural time was $113 \pm 41$ minutes. Acute complications occurred in $4.4 \%$ of the cases 21 . With followup in 1628 patients, AF-free survival was 78.5\% (PAF: 80.6\% vs PersAF 73.3\%; $p<0.001$ ). Left atrium enlargement, female sex, non-PAF, and early recurrence were independent predictors of $A F$ recurrence $(p<$ 0.05).

\section{Conclusions}

RECABA provides detailed insight into current dosing practices and demonstrates cryoablation is safe and effective in real-world use.

\section{Introduction}


Cryoballoon ablation (CBA) is a well-established technique for the treatment of atrial fibrillation (AF), that has been demonstrated to achieve similar safety and efficacy as radiofrequency ablation (RFA) with shorter procedure times. ${ }^{1}$ While the safety and efficacy of AF ablation has been established, it was only until recently that outcomes of AF ablation were examined within large, prospectively enrolled cohorts of patients with AF. The recent report from the Cryo AF Global Registry described primary outcomes of cryoablation used according to local practice around the world. ${ }^{2}$ Country specific evaluations such as the GWTG-AFIB study, the FREEZE Cohort, and the 1STOP registry describe outcomes of cryoablation in the

USA, Germany, and Italy, which have had many years of experience with the cryoablation catheter. ${ }^{3-5}$ Uniquely, a recent report from Miyazaki et al. described the first safety experience with cryoablation in Japan. ${ }^{6} \mathrm{~A}$ comprehensive examination of modern cryoablation outcomes in an early, pan-country experience with cryoballoon ablation has yet to be reported.

The Spanish Catheter Ablation Registry is published annually as the official report of the Spanish Society of Cardiology Working Group on Electrophysiology and reflects general activity in Spanish electrophysiology units but does not describe AF ablation procedures in detail; therefore, patient selection, procedural techniques, and outcomes in real-world use in Spain have not been reported. ${ }^{7}$. The Spanish Registry of Cryoballoon Ablation (RECABA) prospective multicenter observational study aimed to comprehensively evaluate daily clinical practice and the current state of cryoballoon procedures in Spain.

\section{Methods}

\section{Study design and population}

The Registro Español de CrioAblación con BAlón (RECABA; NCT02785991) is an observational, prospective, national, multicenter (supplementary material online, Table S1) study of patients undergoing $\mathrm{CBA}$ for $\mathrm{AF}$ in Spanish centers with experience in the technique (at least 10 procedures per year). Patients were enrolled between September 2016 and January 2019. For inclusion in the study, patients were required to 1) be older than 18 years of age, 2) be eligible for CBA for AF, 3) have a life expectancy greater than 1 year, and 4) sign the informed consent. Patients with both first and repeat procedures were enrolled. AF was classified as paroxysmal (PAF) or persistent atrial fibrillation (PerAF) according to the current ESC guidelines. ${ }^{8}$ Clinical data were collected at the baseline procedure and at the annual followup by the coordinator responsible for each center through an electronic data collection system for clinical trials (eCRF). Data collection, management and quality control are detailed in supplementary material online (S2).

Ethics Committee approval was obtained according to local legislation. The study was conducted in compliance with the most recent version of the Declaration of Helsinki, Spanish laws and regulations (Royal Decree 1090/2015, Royal Decree 1616/2009, Order SAS/3470/2009 of 16 December). This observational study did not require authorization by the Spanish Agency of Medicines and Medical Devices (AEMPS), as stipulated in Royal Decrees 1090/2015 and 1616/2009, since it is a clinical investigation with CE marked medical devices used in accordance with the clinical purpose of the device. 
The study was assessed and approved by the IRB, Comité Ético de Investigación Clínica de Euskadi (CEIC-E) on May 9, 2016, and by the Ethical Committee of Hospital de Mar, Comité Ético de Investigación Clínica del Consorci Mar Parc de Salut de Barcelona (CEIC-Parc De Salut Mar) on June 21, 2016. All patients signed informed consent before inclusion in the registry.

\section{Objective and Endpoints}

The main objective of the RECABA was to report standard clinical practice for PVI using CBA in Spanish hospitals. Standard clinical practice was evaluated by assessing patient characteristics, procedural and CBA techniques, procedure-related complications, the long-term follow-up strategy and efficacy across Spanish centers. The primary endpoint of the study was AF free survival at the 12-month follow-up. Secondary endpoints included the following: (1) a description of baseline characteristics of subjects who underwent CBA, (2) the acute efficacy, safety (the rate of all procedure-related adverse events), and efficiency of the procedure (e.g. electrical PVI, PV potential monitoring, cryoapplications analysis, related complications, and procedural related times), (3) description of the dosing strategies utilized, and (4) identification of the factors that predicted AF recurrence during follow-up.

\section{Cryoballoon ablation procedure}

General pre-ablation management and the CBA procedure were done in accordance with each center's standard protocol for pre-ablation examination, anesthesia and sedation, auxiliary technology for transseptal puncture, anatomical assessment, and phrenic nerve monitoring during right-sided ablation. Nevertheless, the CBA procedure was similar in all hospitals as cryoballoon-over-the-wire techniques were required to follow the internationally accepted techniques described in detail in recent publications and in the supplementary material online (S3). Of note, the following cryo-dosing parameters followed the usual practice of each hospital including: duration of cryoapplications, bonus/non-bonus-freeze strategy, postablation waiting time, and post ablation acute PVI adenosine testing.

\section{Post-ablation management and follow-up}

Oral anticoagulation (OAC) and anti-arrhythmic drug (AAD) management were done in accordance with each center's standard protocol and at the discretion of the cardiologist. Patients could continue taking $A A D$ in the absence of recurrences. Patients were followed with outpatient visits using $24 \mathrm{~h}$ to 30 -day Holter monitoring systems over the 12-month follow-up. Arrhythmia recurrence could also be monitored via electrograms registered in patients with an implanted device prior to (but not after) enrollment in the study. AF recurrence was defined as any AF episode that lasted longer than 30-s accordingly documented (on ECG, Holter monitor, event recording systems or implantable devices). A 3-month blanking period was established, during which detected AF episodes were excluded from the primary endpoint.

\section{Statistical analysis}

Quantitative variables are expressed as mean, median, standard deviation and interquartile range as appropriate. Differences in quantitative variables were evaluated through the student $t$-test for 
independent samples (or analysis of variance depending on the number of groups compared) and between paired variables through the student $t$-test for related samples or the analysis of variance for repeated measures. Categorical data were compared with the chi-square test or Fisher's exact test, depending on the number of categories. The probability of recurrence was predicted using a logistic regression, and a survival analysis predicted the most likely time of recurrence. Univariate models considering each potential predictor were estimated. Subsequently, a multivariate model considering predictors from the baseline visit and procedural characteristics were estimated. An overall multivariate model including significant variables was estimated with non-significant variables as covariates. Time to recurrence was studied using Kaplan-Meier estimates and categorical predictive covariates were included as grouping variables and compared using the log-rank statistic. The Cox-proportional hazard regression method was used to estimate the conditional hazard rate. In order to study AAD use retention at follow-up visits, a logistic loglinear model was estimated, including AF type, AAD use after cryoablation procedure and AF recurrence. Effect confidence intervals and model standardized residuals were obtained. Chisquare for independence was used as test statistic for independence. All analyses were carried out using IBM SPSS v26.0 software. A nominal $5 \%$ significance level was assumed in all analysis and adjustments for multiple comparisons were completed when needed.

\section{Results}

\subsection{Study population}

A total of 1742 patients eligible for CBA for the treatment of paroxysmal $(1238 ; 71.1 \%)$ or persistent (504; 28.9\%) AF were prospectively included from 27 Spanish Centers. The mean number of patients included per center was $64.5 \pm 53.21$, (13 centers enrolled less than 50 patients, 9 enrolled between $51-100,3$ between 101-150 and 2 enrolled more than 150 patients). All but one enrolled patient (who opted out of the procedure after singing informed consent) underwent a CBA. Of all procedures, 1665 (96.6\%) were first CBA procedure and 77 (4.4\%) were repeat ablation procedures.

Clinical characteristics of the study population are shown in Table 1. Most patients were male (68.8\% versus $31.2 \%$; $p 0.001)$, under 65 years old $(70.7 \%)$, and were overweight or obese $(76.1 \%)$. Only 52 patients (3\%) were older than 75 years. One patient was younger than 20 years and 4 patients were older than 80 years of age. The most frequently observed cardiovascular risk factors were hypertension (46.3\%) and dyslipidemia (34.7\%). The population presented a low embolic risk overall (mean $\mathrm{CHA}_{2} \mathrm{DS}_{2}$ VASc index $1.40 \pm 1.28$ ), but $\mathrm{CHA}_{2} \mathrm{DS}_{2}$-VASc was $\geq 2$ in $39.8 \%$ of the cohort. Only $18.9 \%$ of patients had structural heart disease (most frequently tachycardia-related cardiomyopathy and coronary artery disease) and left ventricular ejection fraction was preserved in most patients ( $\geq 50 \%$ in nearly $90 \%$ of the patients). LA was enlarged ( $L A>40 \mathrm{~mm}$ or area $>20 \mathrm{~cm}^{2}$ ) in over half of the patients (most frequently mild). Oral anticoagulation (most frequently direct anticoagulation drugs) and AADs were taken at baseline in $75.5 \%$ of patients. In 148 patients (8.5\%) CBA was used as a first-line treatment. Patients had PAF with a mean time since diagnosis of more than 1 year in $85.6 \%$ of cases. 
Table 1

CLINICAL BASELINE CHARACTERISTICS

\section{Baseline Characteristics}

Age (years) (mean \pm SD (range))

$\leq 35$ years old; $(n, \%)$

$36-64$ years old; $(n, \%)$

65-74 years old; $(n, \%)$

$\geq 75$ years old; $(n, \%)$

Gender, male; n (\%)

$\operatorname{BMI}\left(\mathrm{kg} / \mathrm{m}^{2}\right)$

BMI > 25-30 kg/m² (overweight); $(\mathrm{n}, \%)$

$\mathrm{BMI}>30 \mathrm{~kg} / \mathrm{m}^{2}$ (obesity); (n, \%)

AF type; $n(\%)$

Paroxysmal

Persistent

Prior Catheter Ablation; n (\%)

Previous cryoballoon ablation

Previous radiofrequency ablation

Time since diagnosis < 1 year; $\mathrm{n}(\%)$

Cardiovascular risk factors; $\mathrm{n}$ (\%)

Hypertension

Diabetes mellitus

Smokers

Dyslipidemia

Ischemic stroke/TIA

Vascular disease

Percentage values have been calculated for valid responses and on the total of the sample.

Quantitative data are the mean \pm standard deviation. BMl: body mass index; AF: atrial fibrillation; TIA: transient ischemic attack; $\mathrm{CHA}_{2} \mathrm{DS}_{2} \mathrm{VASc}_{2}$ : Score for $\mathrm{AF}$ and stroke risk; AHS: apnea-hypopnea syndrome; LVEF: left ventricular ejection fraction; AAD: antiarrhythmic drugs; PV: pulmonary veins; LCT: left common trunk; RCT: right common trunk. *High level of exercise defined as $>300$ minutes/week.

\section{$n=1742$}

$58.02 \pm 10.40(20-85)$

49 (2.8)

1183(67.9)

458 (26.3)

52 (3)

$1199(68.8 \%)$

$28.1 \pm 4.26$

$814(46.7)$

512 (29.4)

1237 (71.1\%)

504 (28.9)

77 (4.4)

33 (42.9)

44 (57.1)

245 (14.4)

806 (46.3)

158 (9.1)

224 (13.3)

605 (34.7)

93 (5.3)

97 (5.6) 


\begin{tabular}{|c|c|}
\hline Baseline Characteristics & $\mathrm{n}=1742$ \\
\hline \multicolumn{2}{|l|}{$\mathrm{CHA}_{2} \mathrm{DS}_{2}-\mathrm{VASc} ; \mathrm{n}(\%)$} \\
\hline 0 & $494(28.7)$ \\
\hline 1 & $543(31.5)$ \\
\hline 2 & $346(20.1)$ \\
\hline 3 & $221(12.8)$ \\
\hline 4 & $81(4.7)$ \\
\hline 5 & $31(1.8)$ \\
\hline$>5$ & $6(0.4)$ \\
\hline High Level of exercise*; n (\%) & $80(4.8)$ \\
\hline Sleep Apnea; n (\%) & $206(12.9)$ \\
\hline CPAP AHS treated patients; $\mathrm{n}(\%)$ & $141(68.4)$ \\
\hline Heart disease; n (\%) & $329(18.9)$ \\
\hline Coronary artery disease & $112(34.0)$ \\
\hline Idiopathic dilated cardiomyopathy & $40(12.2)$ \\
\hline Hypertrophic cardiomyopathy & $26(7.9)$ \\
\hline Valvular heart disease & $29(8.8)$ \\
\hline Tachycardia-related cardiomyopathy & $113(34.3)$ \\
\hline Other cardiac disease & $24(7.3)$ \\
\hline \multicolumn{2}{|l|}{ LVEF; n (\%) } \\
\hline$\leq 35 \%$ & $67(4.1)$ \\
\hline $36-50 \%$ & $97(6.0)$ \\
\hline$>50 \%$ & $1456(89.9)$ \\
\hline Left atrium dilatation; n (\%) & $825(51.4)$ \\
\hline Mild (area $20-30 \mathrm{~cm}^{2}$ ) & $384(64.9)$ \\
\hline
\end{tabular}

Percentage values have been calculated for valid responses and on the total of the sample. Quantitative data are the mean \pm standard deviation. BMI: body mass index; AF: atrial fibrillation; TIA: transient ischemic attack; $\mathrm{CHA}_{2} \mathrm{DS}_{2} \mathrm{VASc}_{2}$ : Score for AF and stroke risk; AHS: apnea-hypopnea syndrome; LVEF: left ventricular ejection fraction; $A A D$ : antiarrhythmic drugs; PV: pulmonary veins; LCT: left common trunk; RCT: right common trunk. *High level of exercise defined as $>300$ minutes/week. 


\begin{tabular}{|c|c|}
\hline Baseline Characteristics & $n=1742$ \\
\hline Moderate (area $31-40 \mathrm{~cm}^{2}$ ) & $157(26.5)$ \\
\hline Severe $\left(\right.$ area $\left.>40 \mathrm{~cm}^{2}\right)$ & $51(8.6)$ \\
\hline Left ventricular hypertrophy; $\mathrm{n}(\%)$ & $255(15.8)$ \\
\hline Congestive heart failure; $\mathrm{n}(\%)$ & $141(8.1)$ \\
\hline Other associated arrhythmias; $\mathrm{n}(\%)$ & $383(22.1)$ \\
\hline Atrial tachycardia & $19(1.1)$ \\
\hline Common atrial flutter & $298(17.1)$ \\
\hline Atypical atrial flutter & $26(1.5)$ \\
\hline Other arrhythmias & $40(2.3)$ \\
\hline Implanted cardiac pacemaker; n (\%) & $56(3.2)$ \\
\hline AAD treatment & $1.27 \pm 0.72$ \\
\hline \multicolumn{2}{|l|}{ Prior $A A D$ per patient (mean $\pm S D$ ) } \\
\hline \multicolumn{2}{|l|}{ Previously AAD failed; n (\%) } \\
\hline 0 & $148(8.7)$ \\
\hline 1 & $1068(62.6)$ \\
\hline 2 & $394(23.1)$ \\
\hline$>2$ & $97(5.6)$ \\
\hline Patients taking AAD; $\mathrm{n}(\%)$ & $1307(75.3)$ \\
\hline Flecainide & $781(44.8)$ \\
\hline Amiodarone & $400(23.0)$ \\
\hline Propafenone & $57(3.3)$ \\
\hline Sotalol & $36(2,1)$ \\
\hline Other AAD & $90(5.2)$ \\
\hline Patients taking betablockers; $\mathrm{n}(\%)$ & $1161(67.0)$ \\
\hline
\end{tabular}

Percentage values have been calculated for valid responses and on the total of the sample. Quantitative data are the mean \pm standard deviation. BMI: body mass index; AF: atrial fibrillation; TIA: transient ischemic attack; $\mathrm{CHA}_{2} \mathrm{DS}_{2} \mathrm{VASc}_{2}$ : Score for AF and stroke risk; AHS: apnea-hypopnea syndrome; LVEF: left ventricular ejection fraction; $A A D$ : antiarrhythmic drugs; PV: pulmonary veins; LCT: left common trunk; RCT: right common trunk. *High level of exercise defined as $>300$ minutes/week. 


\begin{tabular}{|c|c|}
\hline Baseline Characteristics & $\mathrm{n}=1742$ \\
\hline Patients taking oral anticoagulation; $\mathrm{n}(\%)$ & $1309(75.5)$ \\
\hline Direct anticoagulation agents & $915(69.9)$ \\
\hline Rivaroxaban & $316(34.5)$ \\
\hline Apixaban & $303(33.1)$ \\
\hline Dabigatran & $186(20.3)$ \\
\hline Edoxaban & $111(12.1)$ \\
\hline \multicolumn{2}{|l|}{ PV anatomy; n (\%) } \\
\hline Left common PV & $277(16.0)$ \\
\hline 2 left PV & $1443(83.6)$ \\
\hline$>2$ left PV & $6(0.3)$ \\
\hline Right common PV & $30(1.7)$ \\
\hline 2 right $P V$ & $1569(91.1)$ \\
\hline$>2$ right $P V$ & $123(7.1)$ \\
\hline \multicolumn{2}{|c|}{$\begin{array}{l}\text { Percentage values have been calculated for valid responses and on the total of the sample. } \\
\text { Quantitative data are the mean } \pm \text { standard deviation. BMI: body mass index; AF: atrial fibrillation; TIA } \\
\text { transient ischemic attack; } \mathrm{CHA}_{2} \mathrm{DS}_{2} \mathrm{VASc}_{2} \text { : Score for AF and stroke risk; AHS: apnea-hypopnea } \\
\text { syndrome; LVEF: left ventricular ejection fraction; } \mathrm{AAD} \text { : antiarrhythmic drugs; PV: pulmonary veins; } \\
\text { LCT: left common trunk; RCT: right common trunk. *High level of exercise defined as }>300 \\
\text { minutes/week. }\end{array}$} \\
\hline
\end{tabular}

\subsection{Procedural characteristics}

Procedure characteristics were analyzed from a total of 1741 CBA procedures (Table 2 ). In nearly $65 \%$ of patients pre-procedural imaging was performed to assess PV anatomy. Most patients (1316 patients; $76.5 \%$ ) had 4 independent PVs. Only $15 \%$ of procedures were performed under general anesthesia. The $28 \mathrm{~mm}$ Arctic Front Advance cryo-balloon was used in $93.8 \%$ of the cases. A bonus freeze strategy was systematically employed in 553 procedures (33.7\%) and was never employed in 679 (41.4\%) procedures. In 407 procedures (24.8\%), a bonus application was administered depending on the perception of the quality of the previous application by the operator. Procedure times (cryotherapy, left atrium, total procedure, and $x$-ray exposure times) are shown in Table 3. Mean number of applications and mean total cryotherapy time was $7.2 \pm 2.67$ and $21.1 \pm 7.80$ minutes per patient, respectively. Patients treated with a no-bonus strategy received significantly fewer (but longer duration) applications, had a shorter time to effect (TTE), and received significantly shorter cryotherapy, LA and fluoroscopy exposure times $(p<$ 0.001); however, total procedure time did not reach statistical significance between no-bonus and bonus strategies $(p=0.07)$. Figure 1 shows the distribution of total cryotherapy $(A)$ and procedural times $(B)$. 
Table 2

PROCEDURAL CHARACTERISTICS

\section{Characteristic}

Pre-procedural imaging; $n$ (\%)

Computerized tomography

Magnetic resonance imaging

Rhythm at time of ablation; $\mathrm{n}(\%)$

Sinus rhythm

Atrial fibrillation

Common atrial flutter

Atypical atrial flutter

Anesthesia and sedation; $\mathrm{n}(\%)$

General anesthesia

Deep sedation

Superficial sedation

Invasive control of blood pressure; n (\%)

Diagnostic catheters (Achieve catheter included); n (\%)

1

2

$>2$

Arctic Front Advance 28 mm; n (\%)

Imaging during transeptal approach; n (\%)

Transesophageal echocardiography

Intracardiac echocardiography

Phrenic nervous monitoring technique; n (\%)

Palpation

Compound motor action potential monitoring

X-ray real time visualization $\mathrm{n}=\mathbf{1 7 4 1}$

$1122(64.4)$

$938(84.0)$

$176(15.8)$

$1275(73.9)$

$424(24.6)$

$33(1.9)$

$7(0.4 \%)$
$261(15.0)$

$537(36.0)$

$953(64.0)$

$710(41.1)$

$298(17.2)$

$811(46.9)$

$621(35.9)$

$1615(93.8)$

$256(14,7)$

$149(58.2)$

$107(41.8)$

$1716(99.1)$

$370(21.4)$

$388(22.4)$

Percentage values have been calculated for valid responses and on the total of the sample.

Quantitative data are the mean \pm standard deviation. 


\begin{tabular}{|ll|}
\hline Characteristic & $\mathrm{n}=\mathbf{1 7 4 1}$ \\
\hline Bonus cryodosing strategy; $\mathrm{n}(\%)$ & $553(33.7)$ \\
\hline Total cryoapplication duration (min) & $21.1 \pm 7.80$ \\
\hline Left atrium time (min) & $74.6 \pm 27.89$ \\
\hline Waiting time post ablation; $\mathrm{n}(\%)$ & $473(28.5)$ \\
\hline Waiting time post ablation (min) & $17.2 \pm 9.55$ \\
\hline Adenosine test; $\mathrm{n}(\%)$ & $71(4.2)$ \\
\hline Cardioversion during ablation; $\mathrm{n}(\%)$ & $542(31.6)$ \\
\hline Cavotricuspid isthmus ablation $\mathrm{n}(\%)$ & $131(7.5)$ \\
\hline Protamine administration per protocol; $\mathrm{n}$ (\%) & $877(51.3)$ \\
\hline Subcutaneous "Z"-stitch; $\mathrm{n}(\%)$ & $1244(72.3)$ \\
\hline Time to discharge (days) & $1.2 \pm 2.01$ \\
\hline $\begin{array}{l}\text { Percentage values have been calculated for valid responses and on the total of the sample. } \\
\text { Quantitative data are the mean } \pm \text { standard deviation. }\end{array}$ \\
\hline
\end{tabular}

\section{Table 3}

PROCEDURAL CHARACTERISTICS BY CRYOABLATION STRATEGY

\begin{tabular}{|lllll|}
\hline Characteristic & Overall & $\begin{array}{l}\text { Bonus } \\
\text { Strategy }\end{array}$ & $\begin{array}{l}\text { Non- Bonus } \\
\text { Strategy }\end{array}$ & $\begin{array}{l}\text { p- } \\
\text { value }\end{array}$ \\
\hline Applications per patient & $7.2 \pm 2.67$ & $8.6 \pm 2.51$ & $6.5 \pm 2.49$ & $<.001$ \\
\hline $\begin{array}{l}\text { Cryotherapy time per application } \\
(\mathrm{s})\end{array}$ & $\begin{array}{l}178.5 \pm \\
57.83\end{array}$ & $173.8 \pm 55.25$ & $181.5 \pm 59.17$ & $<.001$ \\
\hline $\begin{array}{l}\text { Total cryoapplication duration } \\
\text { (min) }\end{array}$ & $21.1 \pm 7.81$ & $24.4 \pm 8.04$ & $19.6 \pm 7.21$ & $<.001$ \\
\hline $\begin{array}{l}\text { Left atrium time (min) } \\
\text { Total procedure time (min) }\end{array}$ & $\begin{array}{l}113.0 \pm \\
41.08\end{array}$ & $115.6 \pm 42.12$ & $111.8 \pm 40.55$ & .072 \\
\hline $\begin{array}{l}\text { Total fluoroscopy time (min) } \\
\text { Intraprocedural complications; }(\mathrm{n},\end{array}$ & $23.9 \pm 1.6$ & $25.0 \pm 15.06$ & $21.1 \pm 17.55$ & $<.001$ \\
\hline \%) & $77.4)$ & $32(5.7)$ & $45(3.7)$ & .085 \\
\hline Quantitative data are expressed as (mean \pm SD) & & & $70.9 \pm 27.38$ & \\
\hline
\end{tabular}

\subsection{Acute procedural outcomes and applications analysis}

A total of 12495 cryoapplications applied to 6715 PVs (277 left and 30 right common trunks) were performed, for a mean of 1.8 applications per vein. At the end of the procedure $97 \%$ of PV were isolated, 
of which $75.8 \%$ were isolated with the first cryoapplication. In $13 \%$ of the cases patients received only 1 application per vein for PVI (4 applications per intervention). The rest of the patients (86.2\%) received more than 1 application in any of the locations. Global and per vein data analysis are summarized in Table 4. Potentials inside the PV could be monitored during ablation in $61 \%$ of PVs with a mean TTE of $52.9 \pm 37.02$ seconds. Figure 1 shows the histograms of the distribution of TTE (C), temperature at PVI (D) and the minimal temperature reached (E). The most frequent cryoapplications duration was 180 or 240 seconds $(F)$. 


\section{Overall}

Total veins $(n)$

Total applications

Applications per vein ( $n$ )

Time per application (s)

PV potentials; $\mathrm{n}(\%)$

TTE (s)

Temperature at $\mathrm{PVI}\left({ }^{\circ} \mathrm{C}\right)$

PVI with 1st application; $(\mathrm{n} / \%)$

Minimum Temperature $\left({ }^{\circ} \mathrm{C}\right)$

Rewarming time (s)

Per vein Analysis

Total veins ( $n$ )

Total applications (n)

Applications per vein

Time per application (s)

PV potentials (\%)

TTE (s)

Temperature at $\mathrm{PVI}\left({ }^{\circ} \mathrm{C}\right)$

PVI with 1st application; $\mathrm{n}(\%) \quad 1487$ (77.67)

Total cryotherapy time (s)

Minimum temperature $\left({ }^{\circ} \mathrm{C}\right)$

$52.1 \pm 34.14$

$-35.2 \pm 9.29$

\begin{tabular}{llll} 
LSPIV & LIPV & RSPV & RIPV \\
\hline 1537 & 1534 & 1691 & 1660 \\
\hline 2828 & 2766 & 3059 & 3148 \\
$1.8 \pm 1.07$ & $1.8 \pm 1.01$ & $1.8 \pm 1.06$ & $1.9 \pm 1.17$ \\
$179.7 \pm 56.14$ & $179.7 \pm$ & $173.6 \pm$ & $179.4 \pm 57.65$ \\
& 59.74 & $58.76^{*}$ &
\end{tabular}

6715

12491

$1.8 \pm 1.11$

$178.5 \pm 57.83$

4098 (61.0)

$52.9 \pm 37.02$

$-33.6 \pm 9.92$

$75.8(6381)$

$-45.4 \pm 8.20$

$38.7 \pm 20.15$

$1110(72.4)^{*} \quad 967(63.2)$ * $997(59.2)^{*} \quad 834(50.6)$ *

$51 \pm 34.3$

$49.0 \pm 38.88$

$59.0 \pm 39.62^{*}$

$-31.3 \pm 9.7^{*}$

$-33.2 \pm 10.78$

$-34.4 \pm 9.43$

1455

(78.35)

$1610(75.0)$

$1552(73.7)^{\llbracket}$

$323.8+$
162.16

$314.0 \pm$

174.86

$340.4 \pm$

193.07

$-43.4 \pm 7.84$

$-47.3 \pm 8.07$

$-44.4 \pm 8.20$

Quantitative data are the mean \pm standard deviation; PV: pulmonary vein, PVI: PV isolation, TTE: time to effect, LSPV: left superior pulmonary vein, LIPV: left inferior pulmonary vein, RSPV: right superior pulmonary vein, RIPV: Right inferior pulmonary vein. * Statistical significance $p<0.001$; $\$ Statistical significance $p<0.01$. 


\section{Overall}

Rewarming time (s)

$43.3 \pm 22.82^{*}$

$36.7 \pm 18.94$

$41.0 \pm 19.63^{*}$

$34.3 \pm 17.80$

Quantitative data are the mean \pm standard deviation; PV: pulmonary vein, PVI: PV isolation, TTE: time to effect, LSPV: left superior pulmonary vein, LIPV: left inferior pulmonary vein, RSPV: right superior pulmonary vein, RIPV: Right inferior pulmonary vein. * Statistical significance $p<0.001$; $\otimes$ Statistical significance $p<0.01$.

\subsection{Periprocedural-related complications}

Periprocedural and procedural-related complications occurred in 120 patients (6.9\%). Acute intraprocedural complications were reported in 77 patients (4.4\%). The most frequent adverse event observed was phrenic nerve injury (49 patients; $3 \%$ ), of which 36 (73.5\%) recovered immediately. All but 3 patients had recovered normal phrenic motility at time of study exit. Other complications occurred in less than $1 \%$ of the patients and are summarized in Table 5 . Notably, $8(0.45 \%)$ patients developed cardiac tamponade, $16(0.97 \%)$ vascular damage ( 1 arteriovenous fistula requiring surgery), $12(0.68 \%)$ transient ST elevation events, and 9 ( 2 intraprocedural and 7 in the 30 -days post ablation) patients had a cerebral stroke or transitory ischemic attack (TIA). In total, major adverse cardiovascular effects (MACE; including stroke/TIA, ST-segment elevation, and cardiac tamponade) occurred in $29(1.6 \%)$ patients. No intraprocedural deaths occurred. 
Table 5

PROCEDURAL RELATED COMPLICATIONS

\begin{tabular}{|ll|}
\hline Complication & $\boldsymbol{n}(\%)^{\mathbb{Z}}$ \\
\hline Total & $120(6.9)$ \\
\hline Acute complication (during procedure) & $49(2.30)$ \\
\hline Phrenic nerve palsy & \\
\hline Resolved by discharge & $36(2.21)$ \\
\hline Unresolved at 12 mo & $10(0.61)$ \\
\hline Cerebral Stroke/TIA & $3(0.18)$ \\
\hline Cardiac tamponade & $2(0.1)$ \\
\hline Transient ST elevation & $8(0.45)$ \\
\hline Hemoptysis & $12(0.68)$ \\
\hline Major bleeding & $1(0.05)$ \\
\hline Other & $2(0.11)$ \\
\hline Subacute complication (up to 30 days post-procedure) & $3(0.22)$ \\
\hline Peripheral vascular damage & $17(0.97)$ \\
\hline Cerebral stroke/TIA & $7(.40)$ \\
\hline Atrio-esophageal fistula & $1(0.05)$ \\
\hline Clinical Gastroparesis & $1(0.05)$ \\
\hline Other & $17(0.97)$ \\
\hline 『: percentage related to the entire population & \\
\hline TIA: transient ischemic attack. & \\
\hline
\end{tabular}

Five deaths $(0.29 \%)$ occurred during follow-up, $2(0.12 \%)$ of which occurred during the first 30 days after the procedure. One death within 30 days was due to a traumatic cerebral hemorrhage and the other due to a mesenteric embolism (the patient was not taking the correct anticoagulant dosage). Three patients died between 140 and 308 days post procedure, one patient died as a result of lung neoplasia, one due to a complication after a left atrial appendage occlusion, and one due to an asystole documented bay emergency service (no more data were collected). In 1 patient an atrio-esophageal fistula occurred during the first month after ablation. The patient presented with global sepsis. This complication was resolved surgically, but the patient suffered serious neurologic damage. No clinical PV stenoses were reported during follow-up. At the end of the study, $95.8 \%$ of the adverse events were resolved. 


\subsection{Follow-up and AF recurrence predictors}

Of the 1742 patients enrolled in the RECABA, 1628 (93.4\%) completed 12-month follow-up (6.54\% lost to follow-up rate, mostly due to restrictions caused by the Covid-19 pandemic) with a median follow-up of 375 (IQR 342-415) days since the index procedure. Arrhythmic event monitoring was conducted with electrocardiography (48.6\%), 24-hour Holter monitoring (39.9\%), 72-hour Holter monitoring (3.6\%), implantable continuous loop recorder (2\%) and non-implantable continuous recorders (3.4\%). The 12month Kaplan-Meier estimate of freedom from AF recurrence after the blanking period was $78.5 \%$ (95\% Cl: 76.3\% - 80.7\%; Fig. 2A). The 12-month estimate of freedom from a $\geq 30$ s recurrence of AF in patients with PAF was superior to those with PerAF (80.6\% Cl: 78.1\%-81.1\% vs 73.3\% Cl: 68.8\%-77.8\% respectively; $\mathrm{p}<0.001$; Fig. 2B).

Baseline patient characteristics and procedural variables were analyzed to identify predictors of AF recurrence (Table 6). Univariate analyses identified the following baseline characteristics predicted AF recurrence: $\geq 65$ years of age, female gender, non-PAF, $\mathrm{CHA}_{2} \mathrm{DS}_{2}-\mathrm{VASc} \geq 2$, no physical activity, structural heart disease, $L V E F \leq 50 \%$, and LA enlargement. The following predictors were found close to the limit of significance: using a bonus strategy $(p=0.098)$ and duration of AF history $>1$ year $(p=0.098)$. A multivariate model identified the following independent predictors of $A F$ recurrence: non-PAF $(O R=1.70$, $p<0.001)$, $L A$ enlargement $(O R=1.35, p=0.017)$, and female gender $(O R=1.33, p=0.039)$. In total, 276 patients $(17 \%)$ had $A F$ recurrence during the blanking period, and $A F$ recurrence during the blanking period was a significant post-procedural predictor of arrhythmia recurrence $(H R=5.98, p<0.001 ; H R=$ $6.06, p<0.001$ for univariate and multivariate analysis respectively). Figure 3 shows Kaplan-Meier free survival curves for highlighted independent and potential predictors AF recurrence. 
Table 6

PREDICTORS OF AF RECURRENCES

\begin{tabular}{|c|c|c|c|c|c|c|}
\hline & \multicolumn{3}{|c|}{ Univariate } & \multicolumn{3}{|c|}{ Multivariate } \\
\hline & OR & OR 95\% Cl & $\mathrm{p}$ & OR & OR $95 \% \mathrm{Cl}$ & $\mathrm{p}$ \\
\hline \multicolumn{7}{|l|}{ Clinical Parameters } \\
\hline Age ( $\geq 65$ years) & 1.46 & $1.16-1.85$ & 0.002 & 1.15 & $0.87-1.51$ & 0.324 \\
\hline Gender (Female) & 1.41 & $1.12-1.76$ & 0.003 & 1.33 & $1.01-1.76$ & 0.039 \\
\hline Persistent AF & 1.77 & $1.40-2.22$ & $<0.001$ & 1.70 & $1.31-2.20$ & $<0.001$ \\
\hline Time since diagnosis ( $>1$ year) & 1.32 & $0.95-1.83$ & 0.098 & 1.38 & $0.98-1.96$ & 0.068 \\
\hline CHA2DS2-VASc $(\geq 2)$ & 1.55 & $1.25-1.93$ & $<0.001$ & 1.18 & $0.89-1.55$ & 0.252 \\
\hline No physical exercise & 1.35 & $1.09-1.68$ & 0.007 & 1.10 & $0.86-1.39$ & 0.449 \\
\hline Structural Heart Disease & 1.36 & $1.04-1.77$ & 0.024 & 1.11 & $0.78-1.56$ & 0.563 \\
\hline Left ejection fraction ( $\leq 50 \%$ ) & 1.42 & $1.00-2.01$ & 0.050 & 1.04 & $0.64-1.70$ & 0.866 \\
\hline Left atrium enlargement & 1.59 & $1.27-1.99$ & $<0.001$ & 1.35 & $1.05-1.73$ & 0.017 \\
\hline Heart failure & 1.41 & $0.97-2.05$ & 0.070 & .87 & $0.53-1.46$ & 0.590 \\
\hline \multicolumn{7}{|l|}{ Procedure Parameters } \\
\hline Bonus strategy & 1.22 & $0.96-1.55$ & 0.098 & 0.87 & $0.68-1.13$ & 0.308 \\
\hline Post-procedure Parameters & $\mathrm{HR}$ & $\mathrm{HR} 95 \% \mathrm{Cl}$ & $\mathrm{p}$ & HR & $\mathrm{HR} 95 \% \mathrm{Cl}$ & $\mathrm{p}$ \\
\hline Early AF Recurrence & 5.98 & $4.96-7.20$ & $<0.001$ & 6.06 & $4.93-7.46$ & $<0.001$ \\
\hline
\end{tabular}

The AF recurrence rate did not differ based on center volume in this study ( $p=n s$ ) (Fig. 4). However, procedure time, fluoroscopy time, cryoablation time, and percentage of isolated veins was improved in more experienced centers, and a bonus cryoapplication strategy was used more often in experienced centers (all $p<0.001)$. The adverse event rate was not different between centers grouped by experience ( $p$ = 0.1) $(\mathrm{S} 4$, Table $\mathrm{S} 2$, supplementary material online).

\subsection{Medications during follow-up}

After CBA, $89.8 \%$ of patients were discharged after 1 day or less (median time to discharge 1 day; mean $1.25 \pm 2.01$ days). Although there were no protocol requirements for $A A D$ usage, medication data were collected. At time of discharge, 1131 patients (65\%) were taking AAD, most frequently Class Ic agents (690 patients; $61.2 \%$ ) and amiodarone (333 patients; $29.5 \%$ ). All patients were taking an OAC agent at discharge $(1270 ; 72.9 \%$ patients taking direct OAC).

Of the 1628 patients who completed follow-up, 1038 were on AADs (63.8\%), with a similar proportion in PAF $(63 \%)$ and PerAF (65.8\%). The percentage of patients free of AF recurrence on AADs at 12-month 
follow-up was also similar between PAF and PerAF groups (29.5\% vs 28.9 respectively, $p=n s)$. Patients discharged under AAD treatment were more likely to continue AADs at the end of follow-up, irrespective of AF recurrence (29\% versus $12 \%$ in the PAF group; $p=0.002)$. Figure 5 displays the cohort according to type of $A F, A A D$ at discharge, recurrences and $A A D$ at the final follow-up.

\section{Discussion}

The RECABA registry is the largest cryoballoon registry in Spain and reflects the standard clinical practice and clinical characteristics of patients treated by CBA for PVI in Spanish hospitals. AF is the most frequently ablated arrhythmia in Spain (27.8\% of all arrhythmias), and last year, $42 \%$ of these procedures were performed with cryoballoon. ${ }^{7}$ The results of RECABA demonstrated acute and subacute complications associated with real-world usage of CBA for treatment of AF were rare (6.9\%), and cryoablation was effective with $79 \%$ freedom from AF at 12-mo when used according to standard clinical practice in 27 unique centers in Spain.

\section{Tailored and Efficient Cryoballoon Ablation Procedure}

Patients in RECABA were mostly young, overweight adult males who presented with PAF without structural heart disease and are consistent with baseline characteristics of patients enrolled in other European, multicenter registries. ${ }^{5,6,9}$. The cryoablation procedure was frequently performed under superficial sedation, which has been reported to result in shorter procedure times without compromising AF recurrences or complication rates. ${ }^{10}$ During recent years, cryothermal dosing protocols have also evolved towards shorter application times based on per-PV TTE with or without bonus applications, and have resulted in more efficient procedure times than fixed cryoapplication times. ${ }^{11-13}$ This trend was also observed in RECABA in which two thirds of the centers systematically used dosing protocols without bonus applications that led to significantly fewer applications per patient and shorter procedure, cryotherapy, and LA times. Centers with more experience applied the bonus strategy to a greater extent; although, there is no clear explanation for this observation. It is possible these centers perceived a greater need for applications as they treated more patients with PerAF; thus, it is important to attempt PV potential monitoring during ablation to tailor dosing to each PV. In the RECABA registry, PV potentials could be seen during ablation in $61 \%$ of the veins and more often in left PVs. These results are similar to contemporary reports, and rates have improved with the introduction of new generations of cryoballoon $^{11,14}$. General procedure times in the RECABA registry, such as cryotherapy, LA and x-ray exposure times were comparable to those obtained in other studies, and they were significantly reduced in non-bonus protocols. 5,7,9,11 These data corroborate a trend toward simplifying and maximizing efficiency of AF ablation procedures.

\section{Antiarrhythmic drugs on long-term outcomes}

There are mixed reports on the influence of short-term use of AADs on long-term outcomes after AF ablation. ${ }^{15-17}$ The European survey of AF ablation practice that included 1300 patients from 72 European 
institutions found that among a patient population that was more than two-thirds PAF, $65 \%$ of patients were discharged post-ablation on an antiarrhythmic medication and $49 \%$ remained on antiarrhythmic medication at 12 months. ${ }^{18}$ The RECABA study observed similar rates of AAD usage after discharge. Although further randomized data are required, patients discharged on AADs were more likely to continue AAD use throughout study follow-up even in the absence of arrhythmia recurrence. The reasons for this may range from a perception of added efficacy of the AAD or its additional effect on the ablation, but more probably it is due to the nature of a registry and lack of strict control in the follow-up. In the group of PerAF, however, the differences were non-significant (Fig. 5).

\section{Predictors of outcomes after cryoablation}

Independent predictors for AF recurrence were female sex, non-PAF, LA enlargement, and early recurrence in blanking period. Non-PAF and LA enlargement are a well-stablished predictors of AF recurrence ${ }^{19}$. In the recent CRYO4PERSISTENT AF Trial ${ }^{20}$, a controlled multicenter study, a single CBA for treatment of PerAF demonstrated $61 \%$ success at 12 months and improved quality of life which is in accordance with the data of the current study. A sub-analysis of the Fire and Ice Trial showed that female sex was associated with an almost $40 \%$ increase in the risk of AF recurrence and cardiovascular rehospitalization after PV isolation ${ }^{21}$. In the RECABA study we found early recurrence in blanking period increased the risk for late AF recurrence 6-fold. Lack of recurrence in the blanking period, however, had less predictive power in the PerAF group. In accordance with this finding, early AF recurrence in blanking period (classically considered as non-valuable and unrelated with late AF recurrence, especially in radiofrequency PV ablations) has been found as a strong predictor of long-term AF recurrence in several recent studies ${ }^{22}$, especially when it occurs in the second or third month after ablation. These data align with some authors suggestion that recurrences during the blanking period should be redefined. ${ }^{23}$

Interestingly, the independent predictors of AF recurrence within the RECABA registry were in general alignment with the findings of large-randomized trials, as well as the safety and efficacy data. Consequently, the RECABA results demonstrate the value of using real-world registries to further validate study findings from randomized controlled trials.

\section{Study Limitations}

The RECABA registry was a multicenter prospective observational registry; therefore, acknowledged limitations include potential bias in patient selection, varied patient management, and the lack of a control group. Nevertheless, possible biases are mitigated by the fact that data were collected prospectively, and research endpoints were pre-specified. As per nature of this project, a standardizedcryoballoon dosing protocol was not implemented. Few patients were implanted with an internal looprecorder; therefore, asymptomatic episodes may have occurred unnoticed, and our success rate may have been over-estimated. Complications that occurred during the ablation procedure may be registered adequately in a registry, but the voluntary nature of data provision may have led to underreporting of procedural complications after the ablation. No recommendations were provided to the participating 
centers in terms of pharmacological treatment following CBA. Thus, the exact temporal sequence of AAD management could not be established, and it is unknown whether AADs were simply continued after the blanking period as per center practice. Despite limitations, this prospective research may provide a representative analysis of the real-world outcomes of CBA in a broad cohort of patients with AF in a large number of centers with different levels of experience.

\section{Conclusions}

Cryoablation was effective ( $79 \%$ freedom from AF at 12 -mo) and safe (6.9\% adverse event rate) when used according to standard clinical practice during the early cryoballoon ablation experience at 27 unique centers in Spain. LA enlargement, non-PAF, female sex and early AF recurrence in the first 3 months independently predicted late AF recurrence.

\section{Perspectives}

\section{Clinical competencies}

- Cryoablation for the treatment of AF performed according to standard clinical practice in 27 centers was safe and effective.

- Cryoballoon ablation is commonly delivered with a tailored, efficient approach.

- Female sex, non-PAF, LA enlargement, and early recurrence in blanking period predicted recurrence during 12-mo follow-up.

\section{Translational Outlook}

Cryoballoon ablation is used to treat a broad group of patients with atrial fibrillation and results in consistent outcomes.

\section{Abbreviations}

AF: atrial fibrillation

PV: pulmonary vein

PVl: pulmonary vein isolation

AAD: antiarrhythmic drugs

CBA: cryoballoon ablation

RFA: radiofrequency ablation

PAF: paroxysmal atrial fibrillation 
PerAF: persistent atrial fibrillation

OAC: oral anticoagulation agents

TIA: transient ischemic attack

MACE: major adverse cardiovascular event

\section{Declarations}

\section{Acknowledgements}

The RECABA investigators want to express their gratitude to the coordinators and electrophysiological teams of all the Spanish centers who have participated in a voluntary and altruistic way in the registry. We also are grateful to Kendra Braegelmann, from Medtronic, and especially with Miguel Angel Ruiz Díaz from Universidad Autónoma (Madrid), for the statistical analysis support.

\section{Competing interests}

Dr. Toquero is member of the Medtronic European advisory board. Dr. Toquero,, Dr. Cózar, Dr. Barrera have received speaker honorarium from Medtronic. Dr. Toquero and Dr. García-Aberola have received educational grant from Medtronic. Dr. Martinez Alday has received speaker honorarium from Medtronic, Boston Scientific and Abbott Laboratories. Dr. Ferrero and Dr. Ruiz have received speaker honorarium from Medtronic and Boston Scientific. Patricia Pascual and Irene Molina are employees of Medtronic Iberia, S.A.

Rest of authors declare no competing interest.

\section{Author contributions statement}

A.F., R.C., A.G., E.V., P.P., I.M., J.M-A have contributed to study design, interpretation of results and manuscript development, A.B., .J.T., J.O., J.S., R.R., P.B., J.M.R, J.M.A have contributed to critical review of the manuscript. All authors have read and approved this manuscript submission and included full disclosures of any potential conflicts of interest.

\section{Data availability statement}

The data that support the findings of this study are available from the corresponding author, A.F., with permission of the Medtronic team upon reasonable request.

\section{References}

1. Kuck KH, Brugada J, Fürnkranz A, et al. Cryoballoon or Radiofrequency Ablation for Paroxysmal Atrial Fibrillation. N Engl J Med. 2016;374: 2235-2245. 
2. Chun, KRJ, Okumura, K, Scazzuso, F, et al Safety and efficacy of cryoballoon ablation for the treatment of paroxysmal and persistent $\mathrm{AF}$ in a real-world global setting: Results from the Cryo AF Global Registry. J Arrhythmia. 2021; 37: 356- 3673.

3. Friedman DJ, Holmes D, Curtis AB et al. Procedure characteristics and outcomes of atrial fibrillation ablation procedures using cryoballoon versus radiofrequency ablation: A report from the GWTG-AFIB registry. J Cardiovasc Electrophysiol. 2021 Feb;32(2):248-259. doi: 10.1111/jce.14858. Epub 2021 Jan 6. PMID: 33368764.

4. Hoffmann E, Straube F, Wegscheider K, et al. Outcomes of cryoballoon or radiofrequency ablation in symptomatic paroxysmal or persistent atrial fibrillation. Europace. 2019; 21: 1313-1324.

5. Tondo C, lacopino S, Pieragnoli P, et al. Pulmonary vein isolation cryoablation for patients with persistent and long-standing persistent atrial fibrillation: Clinical outcomes from the real-world multicenter observational project. Heart Rhythm. 2018; 15: 363-368.

6. Miyazaki S, Kobori A, Sasaki Y, et al. Real-World Safety Profile of Atrial Fibrillation Ablation Using a Second-Generation Cryoballoon in Japan: Insight From a Large-Multicenter Observational Study. JACC Clin Electrophysiol. 2021. doi: 10.1016/j.jacep.2020.11.016.

7. Quesada A, Cózar R, Anguera I. Spanish Catheter Ablation Registry. $19^{\text {th }}$ Official Report of the Heart Rhythm Association of the Spanish Society of Cardiology (2019). Rev Esp Cardiol. 2020. doi10.1016/j.recesp.2020.08.005.

8. Hindricks G, Potpara T, Dagres N, et al. 2020 ESC-Guidelines for the diagnosis and management of atrial fibrillation developed in collaboration with the European Association of Cardio-Thoracic Surgery (EACTS). The Task Force for the diagnosis and management of atrial fibrillation of the European Society of Cardiology (ESC). European Heart Journal. 2020. doi:10.1093/eurheartj/ehaa612.

9. Mörtsell D, Arbelo E, Dagres N, et al. Cryoballoon vs. radiofrequency ablation for atrial fibrillation: a study of outcome and safety based on the ESC-EHRA atrial fibrillation ablation long-term registry and the Swedish catheter ablation registry. Europace. 2019; 21:581-589.

10. Wasserlauf J, Knight BP, Li Z, et al. Moderate Sedation Reduces Lab Time Compared to General Anesthesia during Cryoballoon Ablation for AF Without Compromising Safety or Long-Term Efficacy. Pacing Clin Electrophysiol. 2016; 39:1359-1365.

11. Ferrero-de-Loma-Osorio A, García-Fernández A, Castillo-Castillo J, et al. Time-to-Effect-Based Dosing Strategy for Cryoballoon Ablation in Patients with Paroxysmal Atrial Fibrillation: Results of the plusONE Multicenter Randomized Controlled Noninferiority Trial. Circ Arrhythm Electrophysiol. 2017; 10(12):e005318.

12. Aryana A, Kenigsberg DN, Kowalski M, et al. Verification of a novel atrial fibrillation cryoablation dosing algorithm guided by time-to-pulmonary-vein isolation: Results from the Cryo-DOSING Study (Cryoballoon-ablation DOSING Based on the Assessment of Time-to-Effect and Pulmonary-Vein Isolation Guidance). Heart Rhythm. 2017; 14:1319-1325. 
13. Chun J, Stich M, Fürnkranz A, et al. Individualized cryoballoon energy pulmonary vein isolation guided by real-time pulmonary vein recordings, the randomized ICE-T trial. Heart Rhythm. 2017; 14:495-500.

14. Iacopino S, Pieragnoli P, Arena G, et al. A comparison of acute procedural outcomes within four generations of cryoballoon catheters utilized in the real-world multicenter experience of 1STOP. $J$ Cardiovasc Electrophysiol. 2020; 31: 80-88.

15. Kaitani K, Inoue K, Kobori A, et al. Efficacy of Antiarrhythmic Drugs Short-Term-Use After Catheter Ablation for Atrial Fibrillation (EAST-AF) trial. Eur Heart J. 2016; 37 :610-618.

16. Chen W, Liu H, Ling Z, et al. Efficacy of Short-Term Antiarrhythmic Drugs Use after Catheter Ablation of Atrial Fibrillation-A Systematic Review with Meta-Analyses and Trial Sequential Analyses of Randomized Controlled Trials. PLoS One. 2016. doi: 10.1371/journal.pone.0156121.

17. Duytschaever M, Demolder A, Phlips T, et al. Pulmonary vein isolation with vs. without continued antiarrhythmic drug treatment in subjects with Recurrent Atrial Fibrillation (POWDER AF): results from a multicentre randomized trial. Eur Heart J. 2018; 39: 1429-1437.

18. Arbelo E, Brugada J, Hindricks G, et al. The atrial fibrillation ablation pilot study: a European Survey on Methodology and results of catheter ablation for atrial fibrillation conducted by the European Heart Rhythm Association. Eur Heart J. 2014; 35: 1466-78.

19. Bavishi AA, Kaplan RM, Peigh G, et al. Patient characteristics as predictors of recurrence of atrial fibrillation following cryoballoon ablation. Pacing Clin Electrophysiol. 2019; 42: 694-704.

20. Boveda S, Metzner A, Nguyen DQ, et al. Single-Procedure Outcomes and Quality-of-Life Improvement 12-Months Post-Cryoballoon Ablation in Persistent Atrial Fibrillation: Results From the Multicenter CRYO4PERSISTENT AF Trial. JACC Clin Electrophysiol. 2018 4(11): 1440-1447.

21. Kuck KH, Brugada J, Fürnkranz A, et al. Impact of Female Sex on Clinical Outcomes in the FIRE AND ICE Trial of Catheter Ablation for Atrial Fibrillation. Circulation Arrhtyhmia and Electrophysiology. 2018; 11: e006204.

22. Liu J, Yang H, Liu Y, et al. Early recurrence of atrial tachyarrhythmia during the 90 -day blanking period after cryoballoon ablation in patients with atrial fibrillation: The characteristics and predictive value of early recurrence on long-term outcomes. J Electrocardiol. 2020; 58:46-50.

23. Uetake S, Miyauchi Y, Mitsuishi T, Maruyama M, Seino Y, Shimizu W. Re-definition of Blanking Period in Radiofrequency Catheter Ablation of Atrial Fibrillation in the Contact Force Era. J Cardiovasc Electrophysiol. 2020. doi: 10.1111/jce.14643.

\section{Figures}


A TOTAL CRYOTHERAPY TIME (Minutes)

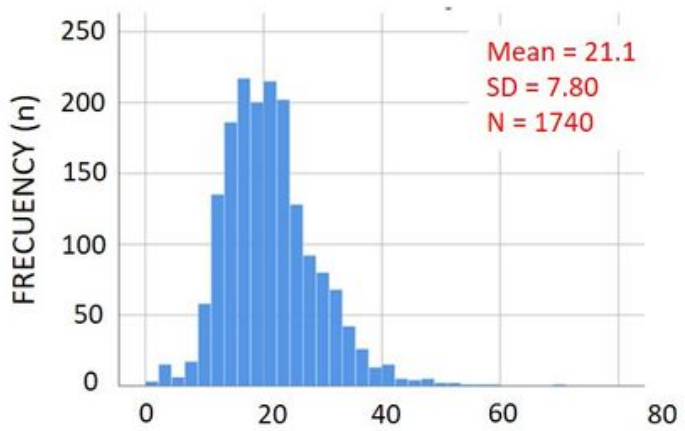

C TTE (seconds)

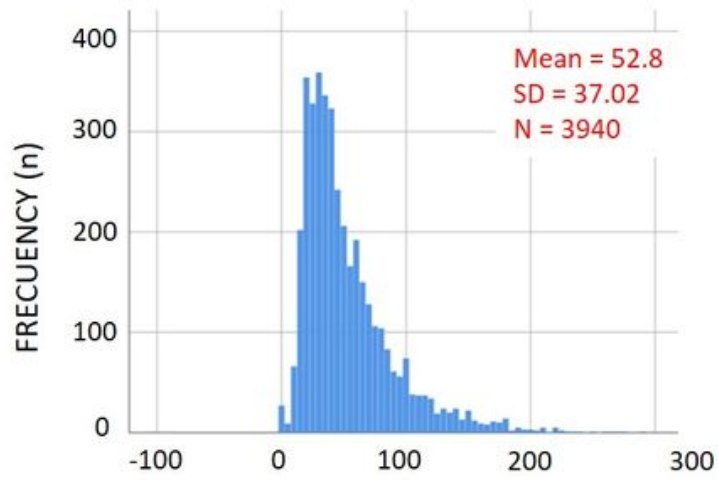

E MINIMAL TEMPERATURE $\mathrm{T}^{0} \mathrm{C}$

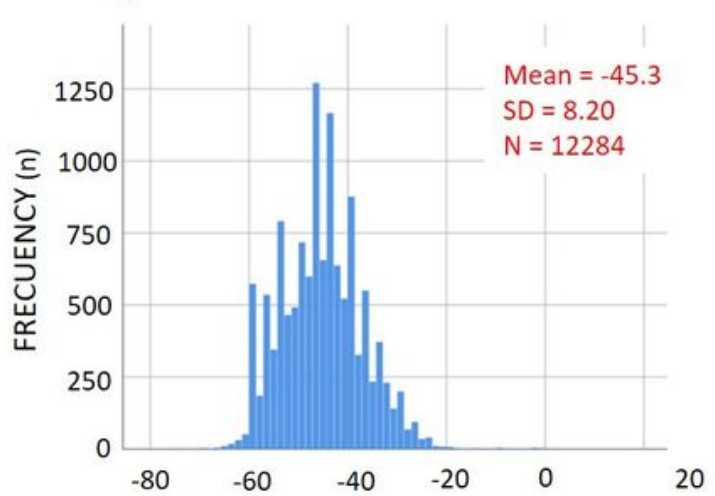

B TOTAL PROCEDURAL TIME (Minutes)

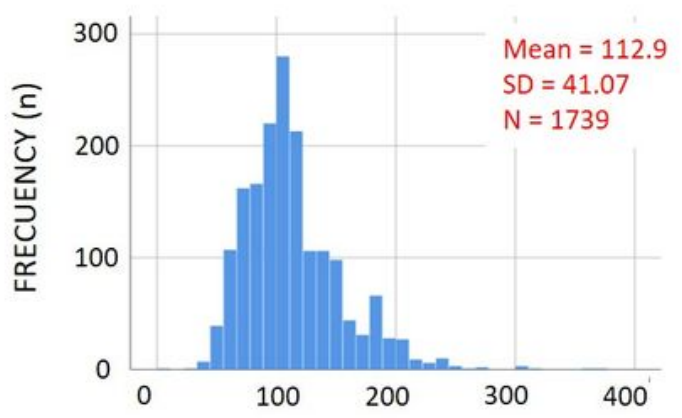

D TEMPERATURE AT PV ISOLATION $\left(T^{0} \mathrm{C}\right)$

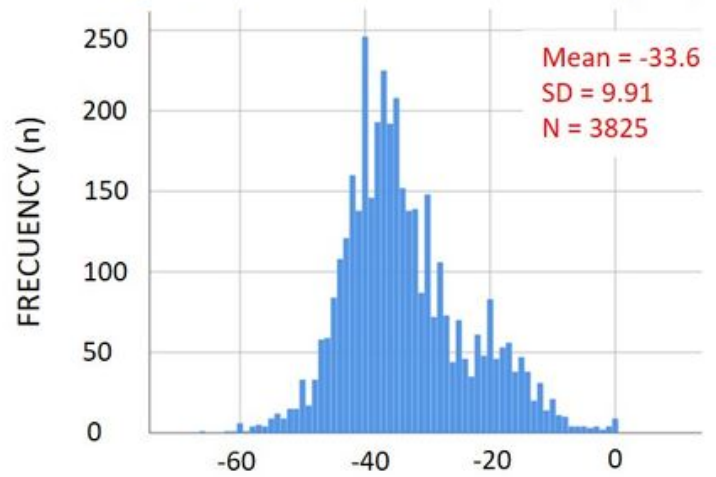

F TIME PER APPLICATION (seconds)

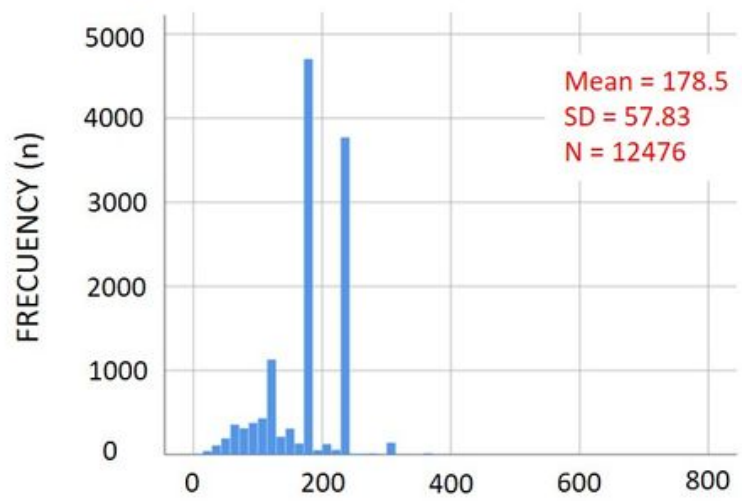

\section{Figure 1}

Distribution of Observed Procedural Characteristics. 


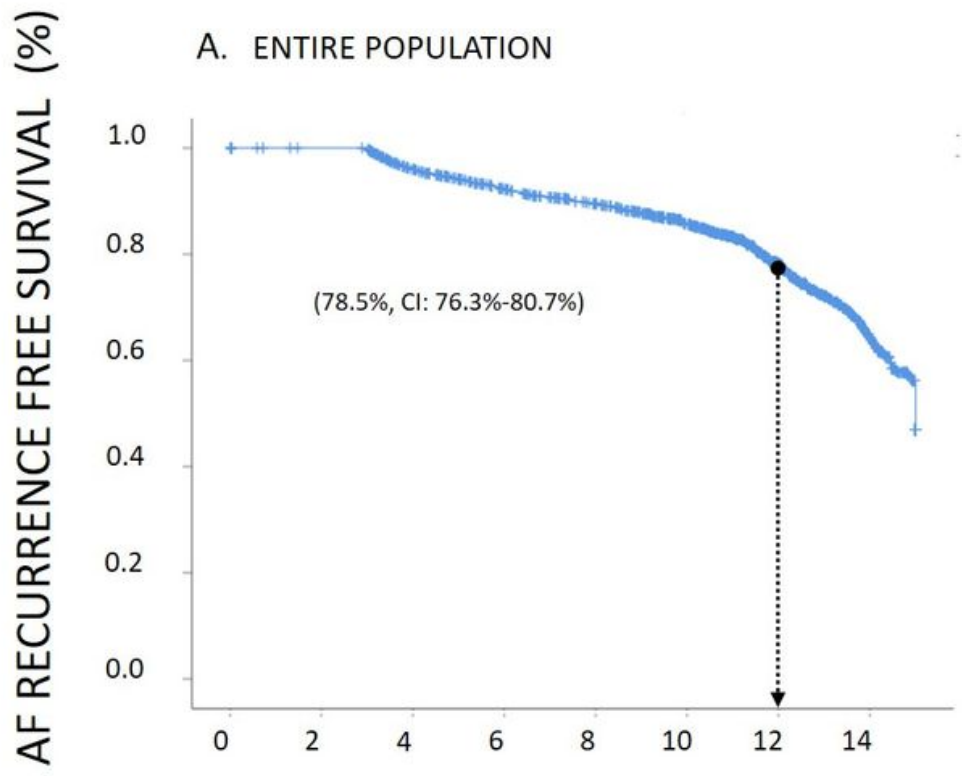

B. PAROXYSMAL AF vS PERSISTENT AF

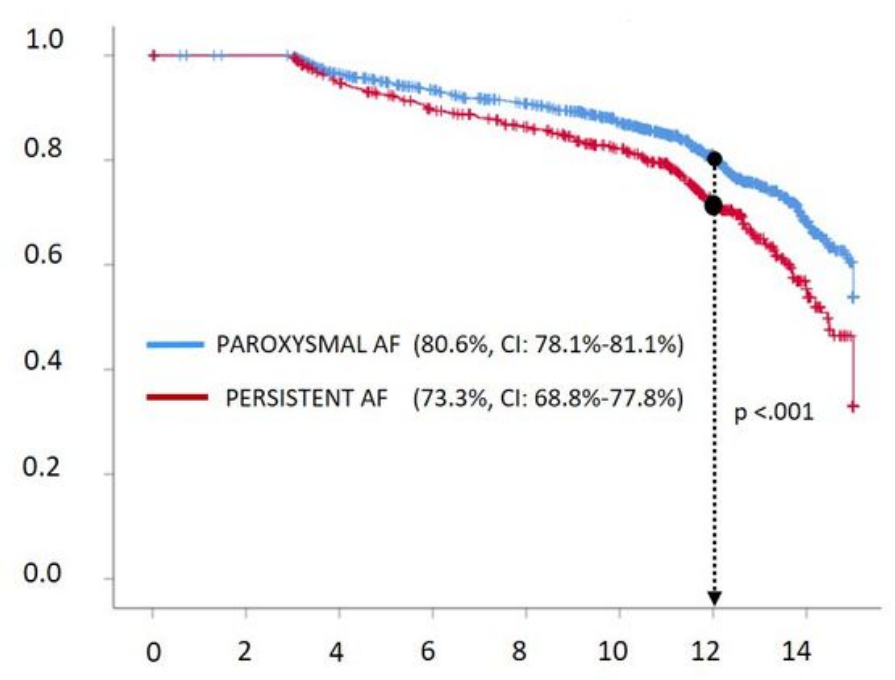

\section{Figure 2}

Freedom from atrial fibrillation over study follow-up. 
A. GENDER: MALE vs FEMALE

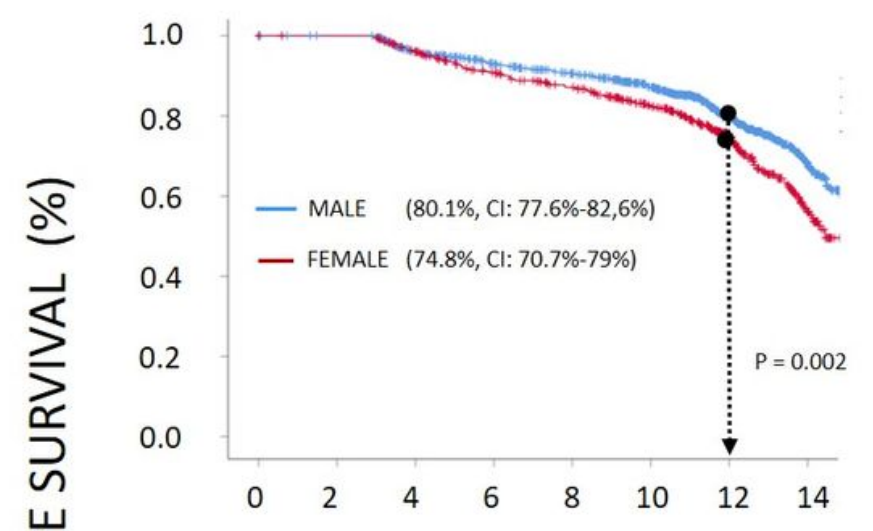

C. BONUS vs NON BONUS STRATEGY

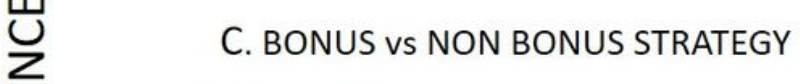

总

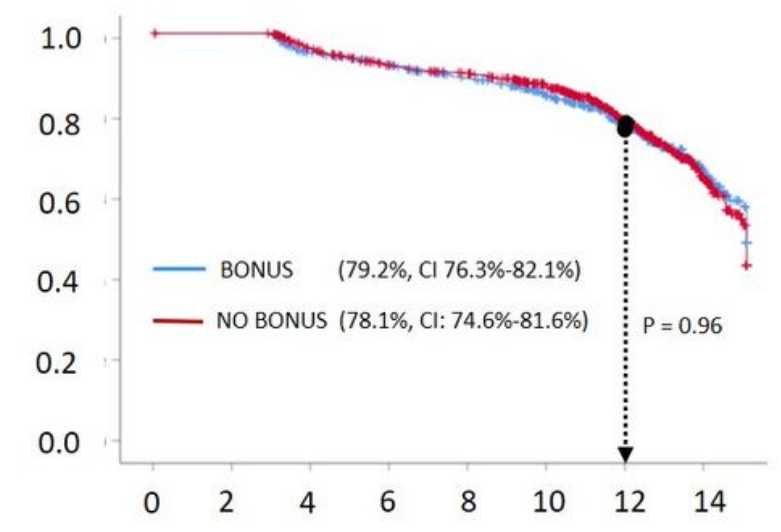

B. LEFT ATRIAL ENLARGEMENT

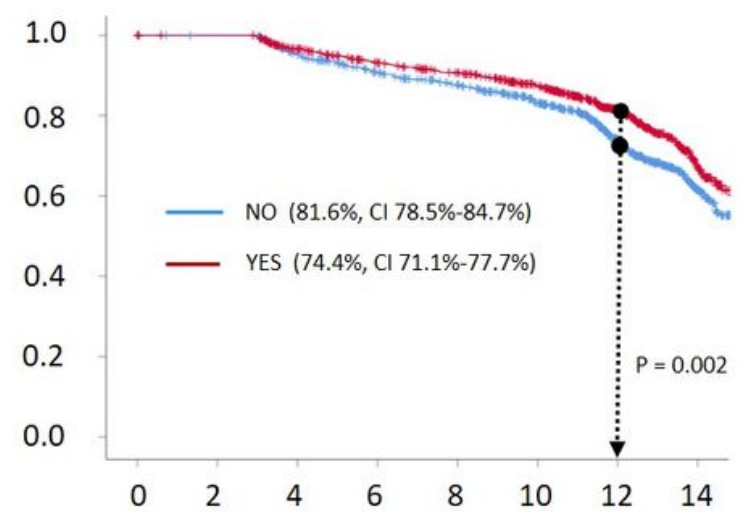

D. RECURRENCE IN BLANKING: PAF vs PERS

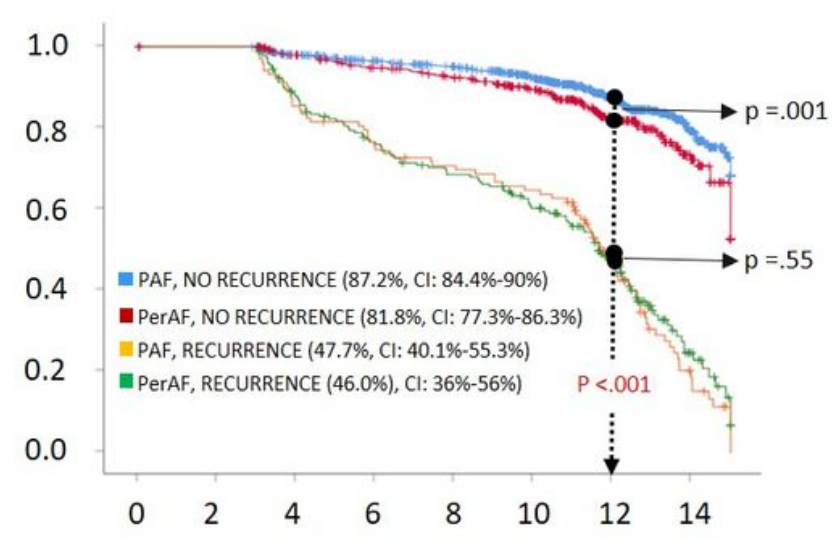

\section{TIME (MONTHS)}

\section{Figure 3}

Analysis of different potential predictors of AF recurrence. 


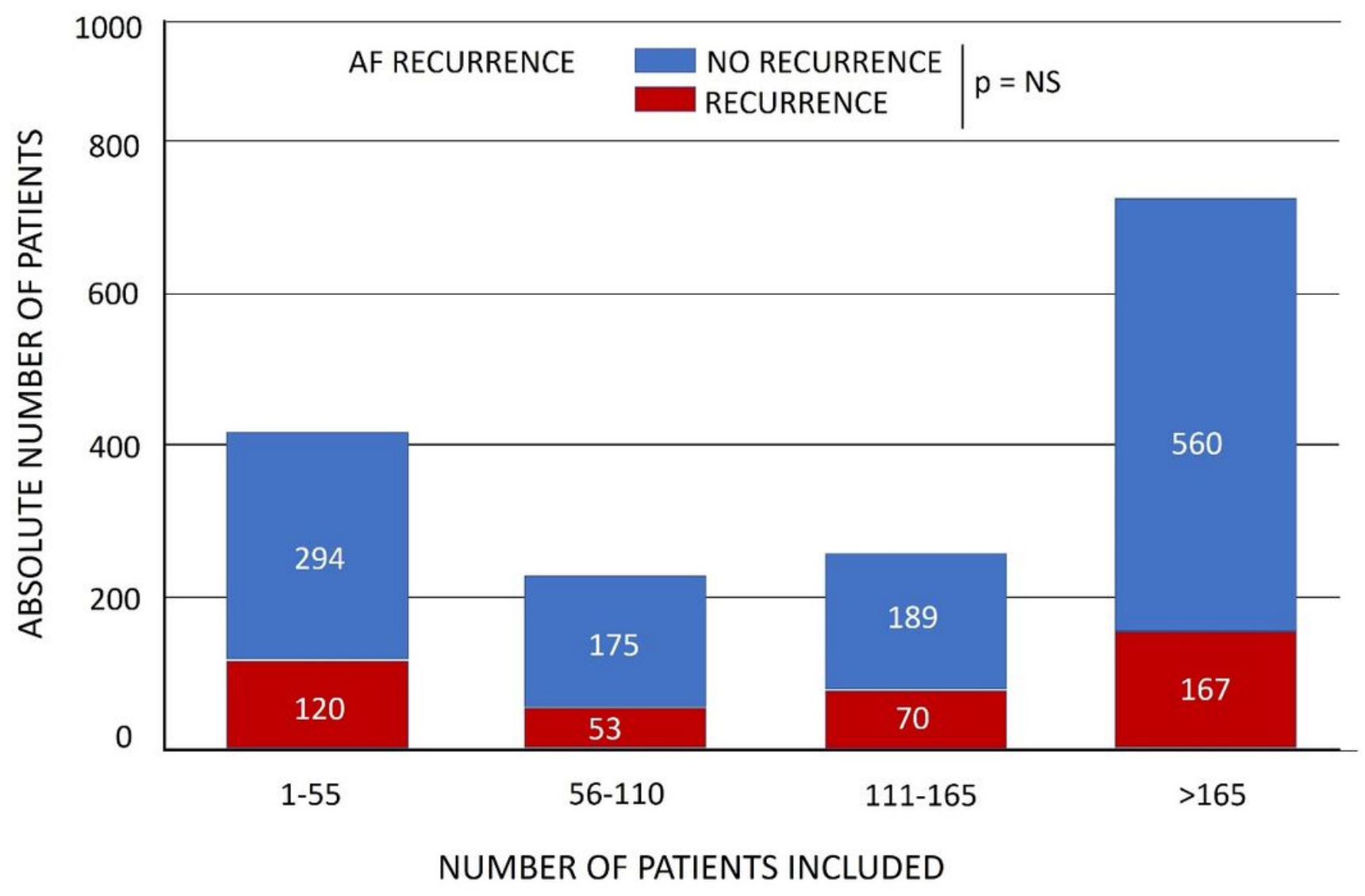

Figure 4

AF recurrence and no-recurrence at 12 months of follow-up (absolute number of patients). 


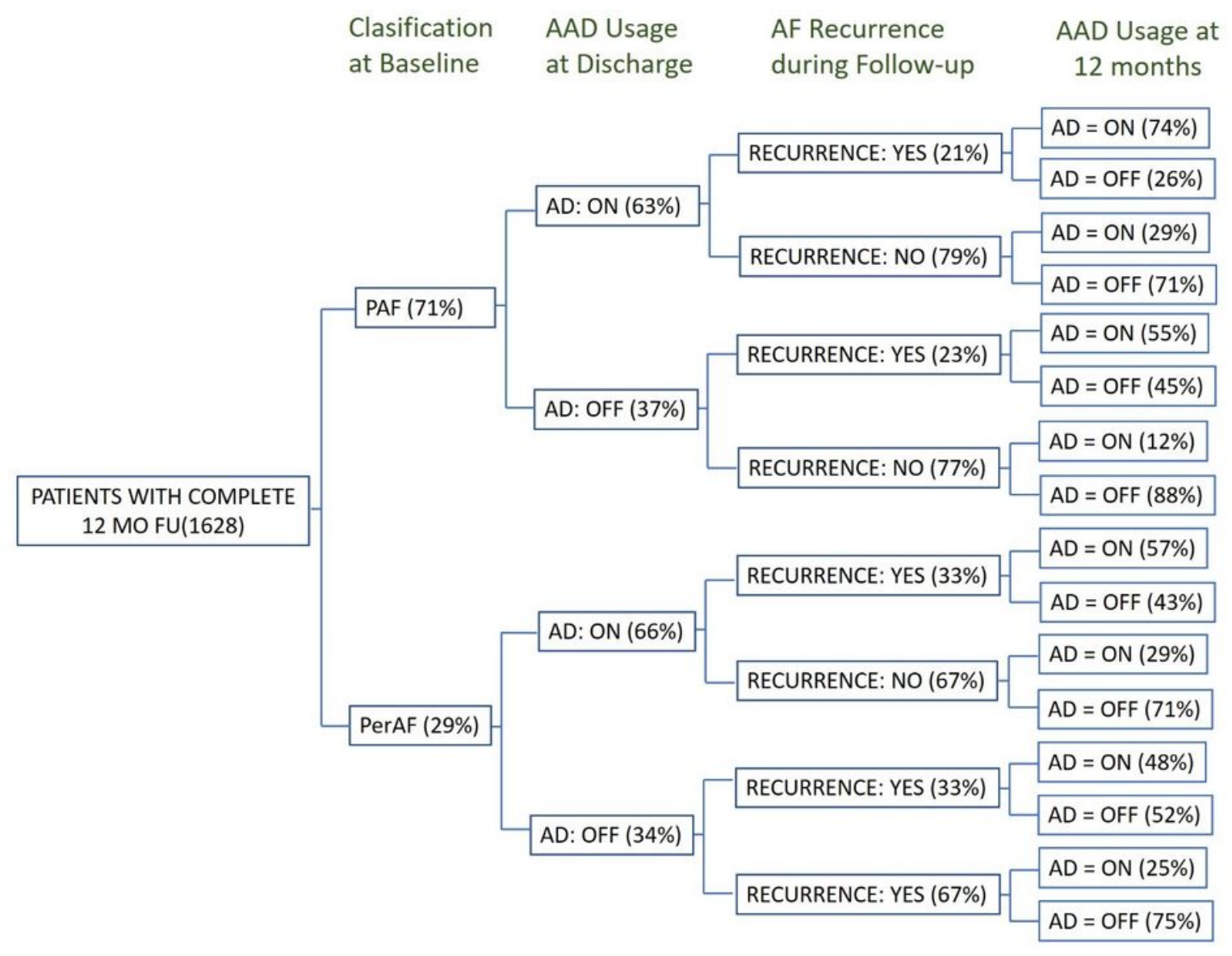

\section{Figure 5}

Flow-chart showing AF recurrence data according to type of $A F, A A D$ at discharge and $A A D$ usage at the final follow-up for the entire population.

\section{Supplementary Files}

This is a list of supplementary files associated with this preprint. Click to download.

- SUPPLEMENTARYMATERIALRecabaScientificReportsClean.docx 